\title{
Editorial
}

\section{Realidades colombianas, una mirada desde la historia, la comunidad y la cátedra}

En el mes de agosto del año 2019, el gobierno de Iván Duque cumplió un año de haber asumido el poder, y en este tiempo son varios los acontecimientos importantes que pueden colaborar a la configuración del panorama nacional en el que se consolida este número de la revisa Ciudad Paz-ando. Sin embargo, puede ser prudente retomar el ambiente nacional que se ha venido tejiendo desde que el ataque terrorista contra la Escuela de Cadetes de Policía General Santander, asumido por el Ejército de Liberación Nacional-ELN, conllevó al cese definitivo de la mesa de negociación que se mantenía con el grupo guerrillero desde el gobierno pasado. En un análisis evaluativo, un poco en retrospectiva y también sobre la marcha, parece que la violencia y el inconformismo siguen imperando en el paisaje nacional a pesar del cambio de gobierno y los nuevos horizontes planteados en el primer semestre del año 2019.

Nos encontramos que, en esta segunda mitad del año, las cosas poco han cambiado, de hecho, pareciera que los sentimientos de inconformidad de los nacionales respecto al desempeño gubernamental, han ido en crescendo a lo largo del año juntándose con los rezagos de los gobiernos anteriores. Si bien han sido varios los acontecimientos cruciales de esta última mitad del año, es prudente mencionar tan sólo algunos, en tanto se consideran incidentes para las interacciones políticas y sociales, tanto en la vida civil común, como en el ejercicio de la política nacional.

Los antecedentes del inconformismo en Colombia respecto al manejo gubernamental pueden remontarse a años, incluso a décadas en el pasado. Sin embargo, serían incluso a décadas en el pasado, sin embargo, serían tres elementos generales, a consideración de quien escribe esta nota, los que llevaron al grueso de la población colombiana a levantarse en protesta el 21 de noviembre del año pasado. En primer lugar, el sentimiento mudo de que la corrupción se mueve sin tapujos ni trabas en medio de los mecanismos que gobiernan el país. Un pensamiento que puede sustentarse en que, si se recuerda bien, Colombia, en el 2016, se consagró como uno de los países más corruptos del mundo según los resultados de la agencia para la Transparencia Internacional en función del Índice de Percepción de Corrupción (IPC).

En segundo lugar, si hay un fenómeno social a nivel nacional, que haya preparado el camino para la explosión de las protestas en el país, este ha sido el asesinato sistemático de líderes y lideresas sociales, como también de defensoras y defensores de los Derechos Humanos, aunado al supuesto poco interés gubernamental por detener tal fenómeno, puesto que para finales del 2019 se registran alrededor de 270 víctimas mortales y otros 600 actores amenazados de muerte.

Finalmente, el inestable panorama político a nivel internacional puede considerarse un tercer factor influyente en la inclinación colombiana al finalizar el 2019. A lo largo de todo el mundo, alrededor de 15 países distintos se suman a la ola mundial de protestas sociales. Algunos ejemplos de esto, que se pueden entender como lejanos en términos geográficos, lo son países o regiones como Francia, Argelia, Sudán, Irak, Irán, Hong Kong o Cataluña. Además, a nivel regional se encuentra que, en Nicaragua, Bolivia, Chile, Ecuador y Haití, se replica la tendencia mundial; los ciudadanos declarados en paro comienzan una serie de protestas a lo largo de semanas, e incluso meses, con el objeto de forzar a sus respectivos gobiernos nacionales a acatar sus diversos pedidos. 
Así las cosas, en Colombia el comité del paro, donde se recogen diversos sectores productivos, poblacionales y otros tantos dentro del marco de la oposición, llaman al paro nacional colombiano y a la salida a las calles en forma de protestas masivas para exigir el cambio estructural de políticas públicas, entre ellas la revisión de las reformas laboral y pensional, además del holding financiero, todos propuestos por el gobierno actual. Es así como como el 21 de noviembre, Colombia entera escucharía, si bien no el primero, el más grande cacerolazo en la historia del país, todo un hito a la organización y empatía ciudadana nacional.

Las marchas no se detienen y para finales del mes de noviembre dejan un saldo de tres personas fallecidas en los enfrentamientos entre los protestantes y los Escuadrones Móviles Antidisturbios (Esmad), entre ellos el joven Dylan Cruz, de 18 años, quien perdió la vida en medio del centro de la capital colombiana; un terrible acontecimiento que se ha convertido en el símbolo de la protesta social y el supuesto uso desmedido de la fuerza por parte de las autoridades nacionales. Finalmente, como resultado de la fuerte presión mediática, a nivel nacional e internacional, sumado a las recomendaciones y llamados de atención al gobierno colombiano, por parte de organizaciones internacionales como la ONU, el presidente Iván Duque decidió convocar, el 2 de diciembre de 2019, a una mesa de negociación al llamado comité del paro.

Por otro lado, no puede dejar de mencionarse el gran triunfo para las mujeres y la población LGBTI+ en las urnas, pues el 27 de octubre Claudia López se convierte en la primera alcaldesa, además abiertamente homosexual, de la capital colombiana. Como se comprenderá, después de lo narrado en este breve texto, López, una vez alcaldesa electa, inicia una fuerte gestión con objeto de apaciguar el convulsionado ambiente que se vive en Bogotá. Ejemplo de esto es, muy en concernencia de la institución cuna de esta revista, la ratificación de la promesa de dialogo para el levantamiento del paro estudiantil, que se conjugó a las protestas nacionales, en la Universidad Distrital Francisco José de Caldas, Institución de educación superior a cago del gobierno de Bogotá. Sin duda, Claudia López representa una alternativa en relación a los gobiernos tradicionales que, vistos a los ojos de los bogotanos, poco han avanzado en materia social, además de una voz firme y decidida hoy en el poder.

Los retos que afrontará la nueva alcaldesa de Bogotá, serán, además del agenciamiento de la situación actual de la capital, la concreción de las diferentes promesas y enfoques políticos que ofreció para la ciudad, como lo son el presupuesto para la educación, la consabida protección de la reserva forestal Van der Hammen, y, como no, la construcción del tan anhelado del metro de Bogotá.

En conclusión, este último tramo del año representa grandes retos en materia política. Es cada vez más clara la postura de exigencia de la ciudadanía en relación a la urgencia de avances sociales, la prudencia en el uso de la fuerza del Estado, el cuidado del medio ambiente, la salud y la educación, entre muchas otras. Por ello, en un momento tan agitado como el presente se hace necesaria, tal vez más que nunca, la reflexión y la investigación con objeto de la producción académica que pueda dar luces de los problemas que tenemos como país, y de las miradas alternas a caminos no muy explorados que pudieran llegar a colaborar en la resolución de conflictos, la reconciliación y la paz nacional, que, como se estipuló en los acuerdos firmados en la Habana en el año 2016, serían las metas máximas de los próximos pasos que daría Colombia como nación.

¿Qué ofrece la edición 12.2 de la revista Ciudad Paz-ando?

Este nuevo número de Ciudad Paz-ando, se desarrolla, a diferencia de su antecesora, en un momento nacional convulso y difícil de comprender. Un momento temporal que exige nuevas miradas al futuro de la política nacional y clama por un compromiso social que se aúne a las necesidades reales de la ciudadanía del país, con el objetivo de no retroceder en el camino hacia la paz. Por ello, en este número recogemos diversos esfuerzos investigativos en harás de colaborar, desde el ámbito académico, a este propósito. El camino de transición a la paz de Colombia, siendo coherente con su historia, oferta increíbles retos y 
riesgos, sin embargo, las investigaciones que acá se presentan tienen la intención de configurar un panorama académico que propenda a la consumación de los objetivos nacionales, desde un enfoque pedagógico, humano y social.

El primer artículo del dossier central de este número, realizado por Hugo Altamirano Acevedo, busca reconocer características importantes de las élites colombianas a partir del análisis del conflicto a lo largo del siglo pasado y la incidencia que han tenido los líderes de izquierda en él. Hace un llamado al reconocimiento de la diversidad política y a la construcción de narrativas honestas y constructivas del pasado nacional. Similar a lo anterior, Liliana del Pilar Escobar Rincón expone diversos elementos teóricos que aportan a la comprensión de la importancia que tiene la inclusión de la historia reciente del país, con el objetivo de la construcción de una memoria histórica que dé cuenta de la verdad, superando el conflicto de lo que se narra y lo que sucedió; proceso que se consolida como un elemento crucial en la configuración de una paz estable y duradera. Finalizando esta pequeña sección, Luz Karime Vanegas Niño y Miguel Ángel Tovar Flórez, complementan la reflexión sobre la importancia de la memoria histórica para la paz, en un artículo donde construyen reflexiones claras sobre la importancia de la constitución de meta-relatos, como un puente entre subjetividades y el reconocimiento del conflicto, para la paz y la reconciliación nacional.

En segunda instancia, Cristhian Adrián García Vergara y Nydia María Rincón Villamizar, presentan una cartografía social del asentamiento humano a la Fortaleza, donde a partir de categorías de análisis como la calidad de vida, la vulnerabilidad y libertades fundamentales, trabajan por el reconocimiento holístico del territorio, evidenciando los recursos activos y las limitaciones sociales con las que conviven sus habitantes. A renglón seguido, Freddy Patiño Montero, establece una serie de conjeturas y evidencias que permiten, por medio del estudio de caso, construir una mirada integral del potencial de la enseñanza de las humanidades en proyectos curriculares enfocados a las ciencias y las tecnologías.

Finalmente, en un artículo de aquellos que develan verdades difíciles de asimilar, Paola Andrea Díaz Bonilla presentan, a partir del partir del testimonio de cuatro jóvenes, diferentes reflexiones que dan cuenta de la cruda realidad de los lugares donde imperan la pobreza y la desigualdad social, espacios idóneos para la vulneración de los Derechos Humanos, en donde los jóvenes que no cuentan con los recursos económicos para la construcción de un proyecto de vida alejado de la violencia, son blanco de un flagelo tan agobiante y escarnecedor como el reclutamiento forzado o la muerte.

Continuando con el análisis académico sobre elementos pertinentes en la construcción de la paz colombiana, en la sección "Voces Otras", Jorge Enrique Blanco, Henry Alexander Castillo, Solangel Aguirre y Johana Alexandra Patiño, presentan un análisis del discurso escolar con el objetivo de determinar los alcances y logros que ha tenido la inclusión de la cátedra de paz, como espacio reglamentario en las escuelas desde La Ley 1732 del 2014 y el Decreto 1038 de 2015. Así, pasamos a la sección "Pensando Regiones", donde Esperanza Milena Torres Madroñero y María Alejandra Botero, postulan la red social como una apuesta epistemológica para el trabajo de reconstrucción relacional, que además se erige como una alternativa comunitaria de carácter contrahegemónico, en oposición a la instauración de la violencia en algunas comunidades de Medellín (Colombia).

En una última sección, este número de Ciudad Paz-ando presenta la reseña del libro $L a$ paz no se rinde: crónicas y memorias de los acuerdos de La Habana, de la escritora, periodista y politóloga vallecaucana Olga Behar Leiser. Esta obra, lanzada al mercado en la Feria Internacional del Libro de Bogotá, el 21 de abril de 2018, presenta una idea optimista y retadora del panorama colombiano, donde los acuerdos de paz firmados en 2016, son el paso de fe que hacía falta para el cambio del imaginario colectivo guerrerista que se ha constituido en la nación en relación al conflicto con las entonces Farc-Ep. Finalmente, para cerrar este interesante número, Wilson Díaz Gamba, director del Director del 
Instituto para la Pedagogía, la Paz y el Conflicto Urbano (Ipazud), y la investigadora Angely Torres Melo, dialogan con estudiantes voceros del comité de Derechos Humanos de la Universidad Distrital Francisco José de Caldas. Una interesante entrevista que da nociones claras sobre cuáles son sus objetivos, alcances, logros y, en definitiva, que es lo que se constituye como la razón de ser de un grupo de tal naturaleza en una realidad capitalina como la actual.

Jairo Andrés Hernández Cubides

EDITOR

Revista CiUdAD PAZ-ANDO 